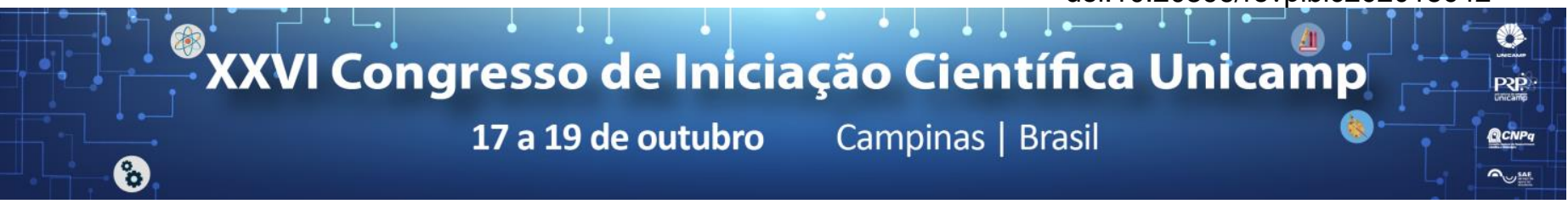

\title{
Cálculo Fracionário e Oscilador Harmônico
}

\section{Izabella Bigosinshi Corrêa*, Mario Conrado Cavichia}

\section{Resumo}

Este trabalho tem o objetivo de estudar os principais conceitos do Cálculo Fracionário e aplicá-los no modelo do oscilador harmônico forçado por meio de equações diferenciais fracionárias. O Cálculo Fracionário é uma ferramenta importante na modelagem matemática de fenômenos físicos e resolução de problemas importantes na engenharia, por poder proporcionar resultados mais próximos dos observados.

\section{Palavras-chave:}

Cálculo Fracionário, Função de Mittag-Leffler, Oscilador Harmônico.

\section{Introdução}

O Cálculo de ordem inteira, ou simplesmente o Cálculo, desenvolveu-se ao longo dos séculos XVIII e XIX, momento em que surgiu a Análise matemática e foram introduzidos formalmente os conceitos de limites, derivada e integral.

A ideia da derivada de ordem não inteira, por sua vez, teve início na troca de correspondências entre Leibniz e L'Hôpital em 1695. Atribui-se a essa discussão os primórdios do Cálculo Fracionário (ou Cálculo de ordem não inteira), que envolve potências de números reais ou complexos em operadores diferenciais.

As integrais fracionárias foram definidas ao longo da história por diferentes estudiosos. A integral fracionária de Riemann-Liouville, a integral fracionária de Weyl e a integral fracionária de Liouville são alguns exemplos.

As derivadas fracionárias, assim como as integrais, possuem diferentes definições. As derivadas de Caputo e de Riemann-Liouville são alguns exemplos.

A fim de analisar uma das aplicações do cálculo fracionário, foi estudada a equação diferencial que representa o oscilador harmônico forçado.

A seguir é apresentada a equação diferencial fracionária que representa o oscilador harmônico forçado sem amortecimento:

$$
D^{\alpha} x(t)+\omega^{\alpha} x(t)=f(t),
$$

sendo $D^{\alpha}$ a derivada de ordem $1<\alpha \leq 2$ no sentido de Caputo, $\omega=\sqrt{k / m}$ a frequência angular, $x(t) \quad 0$ deslocamento em um tempo $t, f(t)=q(t) / m, q(t)$ a força externa que age no sistema e $m$ a massa.

\section{Resultados e Discussão}

Para obter a solução $x(t)$ da equação diferencial fracionária, que representa o oscilador harmônico forçado e sem amortecimento, utilizou-se a metodologia da transformada de Laplace e transformada de Laplace inversa. A solução é dada em termos da função de Mittag-Leffler, $E$, de um e dois parâmetros, a qual é uma generalização da função exponencial. A solução é apresentada a seguir:

$$
\begin{gathered}
x(t)=\int_{0}^{\pi}(t-\tau)^{\alpha-1} E_{\alpha_{\alpha} \alpha}\left(-\omega^{\alpha}(t-\tau)^{\alpha}\right) f(\tau) d \tau+ \\
+x^{(0)} E_{\alpha}\left(-\omega^{\alpha} t^{\alpha}\right)+x^{s}(0) t E_{\alpha_{\alpha} 2}\left(-\omega^{\alpha} t^{\alpha}\right)
\end{gathered}
$$

Para as condições iniciais $x(0)=0$ e $x^{\prime}(0)=0, f(t)=10$ e $\omega=1$, tem-se o gráfico apresentado na Figura 1 , com a ordem da derivada, $\alpha$, variando de 1,2 a 2 .

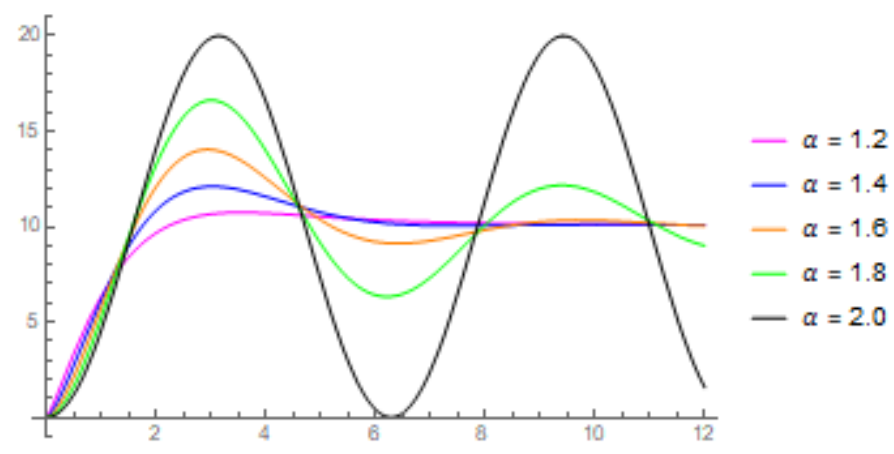

Figura 1. Solução $x(t)$ para o oscilador harmônico forçado.

Nota-se que para $\alpha=2$ recupera-se a solução da equação diferencial ordinária que representa o oscilador harmônico.

\section{Conclusões}

Através dos resultados expostos, é possível perceber que o cálculo fracionário é uma ferramenta matemática de grande importância para a modelagem de fenômenos físicos, devido à variação da ordem $\alpha$ das derivadas que compõe a equação diferencial. Isso permite obter novos resultados a partir de modelos consagrados na engenharia, como a viga de Euler-Bernoulli.

\section{Agradecimentos}

Agradeço ao PIBIC/CNPq pela concessão da bolsa de Iniciação Científica e ao Prof. Dr. Mário Conrado Cavichia pela orientação.

CAMARGO, Rubens de Figueiredo; OLIVEIRA, Edmundo Capelas. Cálculo Fracionário. 1 ed. São Paulo, SP: Editora da Física, 2015.

BOYCE, William E. Equações diferenciais elementares e problemas de valores de contorno. Coautoria de Richard C. DiPrima. 8. ed. Rio de Janeiro, RJ: Livros Técnicos e Científicos, 2006. 